\title{
Effects of short-term aerobic, resistance and combined exercises on the lipid profiles and quality of life in overweight individuals with moderate hemophilia A: A randomized controlled trial
}

\author{
Behrouz Parhampour ${ }^{1}$, Mehdi Dadgoo ${ }^{1 *}$ (D) Giti Torkaman ${ }^{2}$, Roya Ravanbod $^{2}$, Tina Delsouz Bahri ${ }^{3}$, Mohammad Jazebi ${ }^{4}$, \\ Seyed Mehdi Mohsenipour ${ }^{1}$, Behnoosh Vasaghi-Gharamaleki ${ }^{5}$ \\ Received: 21 Jan 2020 \\ Published: 1 Jun 2021
}

Abstract

Background: Overweight is related to increased risks of cardiovascular diseases and dyslipidemia, and reduced quality of life (QOL). Exercise training improves QOL and modifies cardiovascular risk factors and lipid profile. The present study was conducted to compare three types of exercise in terms of their short term effects on QOL and lipid profile in overweight individuals with moderate hemophilia A (IWMHA).

Methods: This study was a randomized, controlled, assessor-blinded trial (IRCT20180128038541N1). Sixty IWMHA with a body mass index (BMI) of $25-30 \mathrm{~kg} / \mathrm{m} 2$ and a mean age of 35-55 years were randomly assigned to four groups of 15 , namely aerobic training (AT), resistance training (RT), combined training (CT) and control. The intervention groups participated in 45-minute exercises three days a week for six weeks. The 36-item short-form health survey (SF-36) was used to measure QOL. Total cholesterol (TC), triglycerides (TG), low-density lipoprotein cholesterol (LDL-C), high-density lipoprotein cholesterol (HDL-C), waist-to-hip ratio (WHR), and waist circumference (WC) were measured before and after six weeks of training. For the data analysis using SPSS version 20, the ANCOVA was used to determine the differences among the four groups.

Results: A significant decrease was observed in the intervention groups compared to the control group in terms of weight, BMI, LDL-C, TC, WHR, and WC $(\mathrm{p}<0.05)$. Significant increase was observed in HDL-C and SF-36 subscales in the intervention groups compared to the control group $(p<0.001)$. There was no significant difference among the intervention groups $(p>0.05)$. In comparison with the control group, more significant improvement was observed in the TC, TG, LDL-C, HDL-L, and SF-36 subscales in the CT group compared to the RT and AT groups.

Conclusion: CT was the most effective training method in improving lipid profile and QOL in overweight IWMHA.

Keywords: Hemophilia A, Overweight, Lipid Profile, Combined Training, Quality of Life

Conflicts of Interest: None declared

Funding: Iran University of Medical Sciences

\section{*This work has been published under CC BY-NC-SA 1.0 license. \\ Copyright $\odot$ Iran University of Medical Sciences}

Cite this article as: Parhampour B, Dadgoo M, Torkaman G, Ravanbod R, Delsouz Bahri T, Jazebi M, Mohsenipour SM, Vasaghi-Gharamaleki B. Effects of short-term aerobic, resistance and combined exercises on the lipid profiles and quality of life in overweight individuals with moderate hemophilia A: A randomized controlled trial. Med J Islam Repub Iran. 2021 (1 Jun);35:70. https://doi.org/10.47176/mjiri.35.70

\section{Introduction}

As an X-linked bleeding disorder, hemophilia A is asso-

\section{Corresponding author: Dr Mehdi Dadgoo, dadgoo.m@iums.ac.ir}

1. Rehabilitation Research Center, Department of Physiotherapy, School of Rehabilitation Sciences, Iran University of Medical Sciences, Tehran, Iran

2. Department of Physical Therapy, Faculty of Medical Sciences,Tarbiat Modares University, Tehran, Iran

3. Department of Microbiology, Faculty of Medicine, Shahid Beheshti University of Medical Sciences, Tehran, Iran

4. Iranian Comprehensive Hemophilia Care Center, Tehran, Iran

5. Rehabilitation Research Center, Department of Rehabilitation Basic Sciences, School of Rehabilitation Sciences, Iran University of Medical Sciences, Tehran, Iran ciated with coagulation factor VIII deficiency (1). An in-

\section{$\uparrow$ What is "already known" in this topic:}

Cardiovascular risk factors such as obesity have become increasingly prevalent in IWH, and weight control can cause more effective conservation of normal joint function and improved quality of life. Exercise training is the best primary and secondary prevention of chronic diseases, especially CVD, obesity, and lipid disorders in different ages.

\section{$\rightarrow$ What this article adds:}

In overweight patients with moderate hemophilia $\mathrm{A}$, all types of exercise can decrease the LDL-C, TC, TG, and increase HDL-C and quality of life. Combination of aerobic and resistance training has the most significant desirablle changes in lipid profile and quality of life and thus can be used for disorders associated with sedentary lifestyle in IWH. 
crease from 30 to $60-70$ years has been reported in the life expectancy of individuals with hemophilia (IWH) in developed countries (2). Thus, age-related complications, especially cardiovascula $\mathrm{r}$ risk factors such as obesity, have become increasingly prevalent in IWH (3). Hemophilia causes a negative perception of the quality of life (QOL) due to diminished independence, pain, and fear of the future (4). Restricted physical activity because of fear of bleeding also reduces QOL in IWH (4). Given the pathophysiological synergy between hemophilia and obesity, controlling weight in IWH can more effectively conserve a normal joint function and improve QOL. Obesity disrupts physical activity and increases the frequency of bleeding and the administration of factor VIII in hemophiliacs (5). Overweightness thus has an indirect role in the poor QOL experienced by IWH (4). Research suggests body mass index (BMI) is positively correlated to total cholesterol (TC), low-density lipoprotein cholesterol (LDL-C), and triglycerides (TG), and negatively correlated with high-density lipoprotein cholesterol (HDL-C) (6). This correlation between BMI and the levels of lipoproteins, especially LDL-C, is a significant contributing risk factor of cardiovascular disease (CVD) in obese individuals (7). High levels of TC and LDL-C have also been well documented to play a vital role in developing arteriosclerosis and coronary artery disease (8). Research suggests that exercise training is the best primary and secondary prevention of chronic diseases, especially CVD, obesity, and lipid disorders in men and women of different ages (9). Aerobic training (AT) and resistance training (RT) are both recommended as significant therapeutic modalities to modify cardiovascular risk factors (10). Combined training (CT) is defined as a combination of simultaneous AT and RT programs in a regular exercise routine (10). Exercise training may increase the risk of injury, particularly re-bleeding in IWH. Exercise training improves joint function in IWH by increasing their muscle function, muscle strength, and joint stability (10).

Decreased levels of LDL-C, TC, and TG and increased levels of HDL-C were reported after resistance, aerobic, and combined exercises in the literature (11-19). To the best of the authors' knowledge, exercise training has not been compared yet in terms of their effect on lipid profile in IWH. The present research was therefore performed to investigate the effect of six weeks of aerobic, resistance, and combined training on QOL and the serum levels of TC, TG, LDL-C, and HDL-C in overweight IWMHA.

\section{Methods}

\section{Study design and sampling}

This study was a randomized controlled trial. The study was registered in the Iranian Registry of Clinical Trials (IRCT20180128038541N1). Written informed consent forms were signed by all the participants. This study was conducted by a team of specialists with 15-20 years of clinical and research experience of IWH. After examination by the hemophilia specialists and physiotherapists familiar with the treatment of IWH, all volunteers were allowed to participate in the study if they met the inclusion criteria. The eligible candidates comprised non- smoking IWMHA aged 35-55 years with no history of an inhibitor, a BMI of $25-30 \mathrm{~kg} / \mathrm{m}^{2}$, a total hemophilia joint health score (HJHS) of at most ten without diabetes mellitus, hepatitis $\mathrm{B}$ or $\mathrm{C}$, human immunodeficiency virus (HIV) and factor VIII prophyllaxis before and during the treatment protocol and under nnusculoskeletal assessments in the Iranian Comprehensive Hemophilia Care Center (ICHCC). The exclusion criteria consisted of clinical symptoms of active bleeding, uncontrolled hypertension, i.e., a systolic blood pressure (SBP) of over $140 \mathrm{mmHg}$ and diastolic blood pressure (DBP) of over $100 \mathrm{mmHg}$, and performing regular physical activities within the previous six months. Sports facility in Tarbiat Modares University was employed for the exercises from March $30^{\text {th }}$ and May $31^{\text {st }}$, 2018. Given the mean \pm standard deviation of TC between the combined and control groups after the intervention in a study by Azarbayjani et al. (13), and considering 95\% confidence interval and $80 \%$ study power, the sample size was determined as 15 participants in each group. According to Figure 1, all the participants were randomly assigned to four groups, namely AT, RT, CT, and control. Randomization was performed by an external observer using closed envelopes in blocks of eight, in each block two persons were randomly assigned to one of the groups. The laboratory technicians and the person in charge of marker measurements were blinded to the grouping. In contrast, the physiotherapist in charge of therapeutic exercises, who was to complete the joint health questionnaire, was not blinded to the grouping.

\section{Exercise protocols}

The participants completed eighteen 45-minute training sessions three times a week, every other day, within six weeks. Based on the findings of a previously-conducted study, the intervention duration was considered six weeks to prevent potential complications (20). Before beginning the exercise protocol, the RT group was assigned a 10repetition maximum $(10 \mathrm{RM})$ for resistance exercises (RE), and a 1-repetition maximum (1RM) was determined using $10 \mathrm{RM}=75 \%$ 1RM (20). The number of repetitions of RE was ten repetitions per odd weeks and twelve repetitions per even weeks. Resisttance was fixed at $65 \%$ of $1 \mathrm{RM}$ in the first two weeks, $70 \%$ of $1 \mathrm{RM}$ in the second two weeks, and $75 \%$ of $1 \mathrm{RM}$ in the third (10). Twelve repetitions of every exercise were performed in the $2^{\text {nd }}, 4^{\text {th }}$ and, $6^{\text {th }}$ weeks and ten repetitions in the $1^{\text {st }}, 3^{\text {rd }}$ and, $5^{\text {th }}$. Ten-second breaks were considered between the repetitions and 1-2 minute breaks between the muscle group exercises (Fig. 2). Free weights, barbells, and dumbbells were employed to perform $\mathrm{RE}$, including the squat, the chest press, the shoulder press, the leg press, calf raise, the knee flexion, and the leg extension. Moreover, exercises for the lower and upper extremities were bilaterally performed. In all the sessions, core exercises first performed in all the sessions were followed by upper and lower limb exercises $(21,22)$. RE was designed based on ACSM's (American College of Sports Medicine) recommendation (large before small muscle group exercises, and multiplejoint exercises before single-joint exercises), and taking into account musculoskeletal bleeding pattern in $\mathrm{PWH}$ 


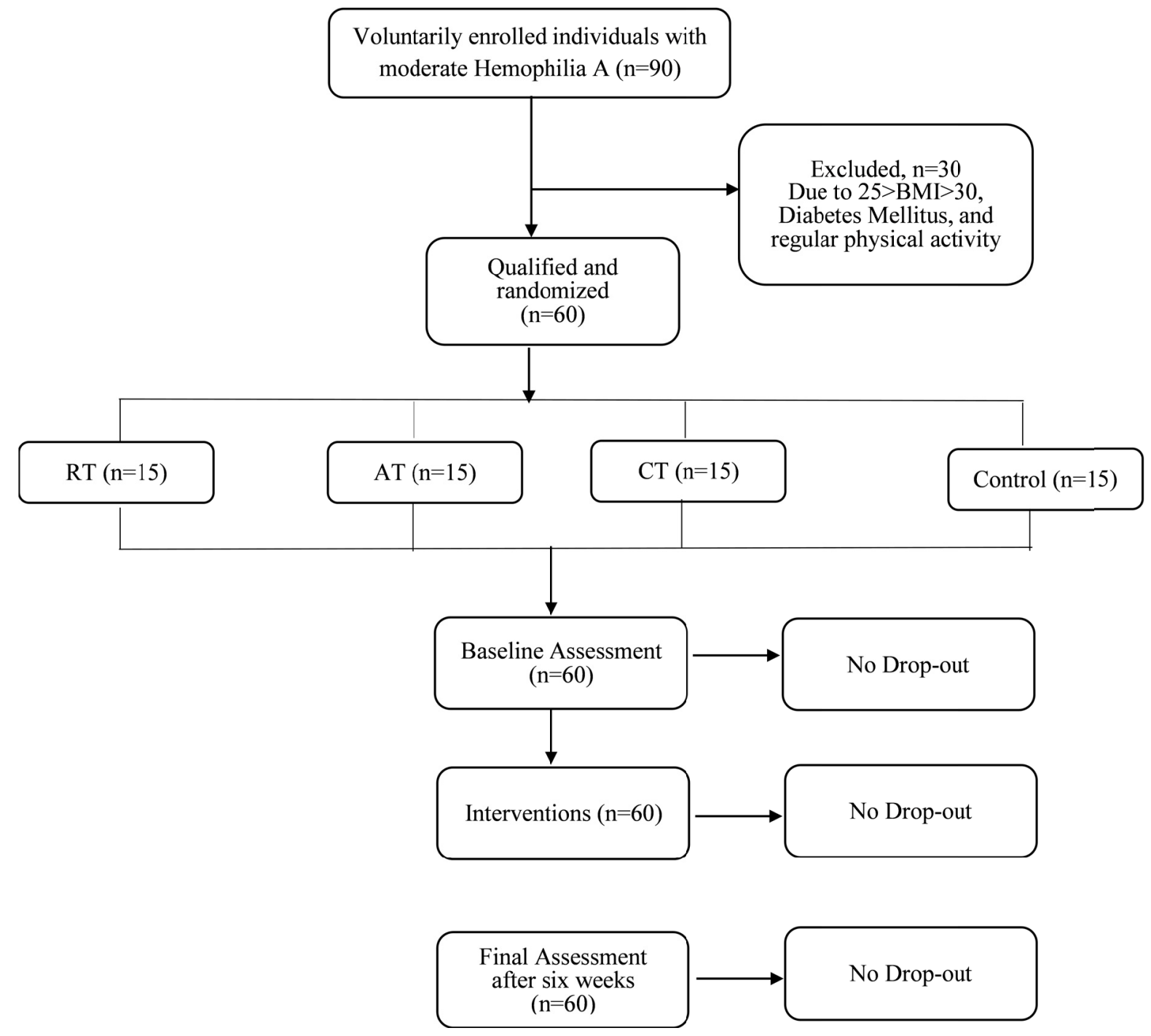

Fig. 1. The study design and the flow of participants. bmi, body mass index; rt, resistance training; at, aerobic training; ct, combined training

(10). During each training session, the participants were asked to perform ten minutes of global and segmentary stretching (as warm up) before starting the exercise protocol $(10,20)$. The participants in the AT group performed a 20-minute aerobic treadmill exercise and ergometer cycling for 20 minutes with a two-minute rest in between. The 220-age equation was used to adjust the aerobic exercise intensity at $65 \%$ of maximum heart rate (MHR) during the first two weeks, $70 \%$ of MHR during the second two weeks and $75 \%$ during the third $(10,23)$. Every step respectively involved a warm-up, exercising at a constant workload, and a cool-down. The aerobic exercise comprised a 5-minute warm-up followed by a 12-minute exercise and a 3-minute cool-down (Fig. 3). The participants in the CT group first performed aerobic exercises for 20 to 25 minutes, took a two-minute break, and then performed resistance training for 20 minutes. Although the intensity of RE was the same in both the CT group and the RT group, the $\mathrm{CT}$ group performed six repetitions of every exercise in the $2^{\text {nd }}, 4^{\text {th }}$, and $6^{\text {th }}$ weeks of training and five repetitions in the $1^{\text {st }}, 3^{\text {rd }}$, and $5^{\text {th }}$ weeks. Every session of aerobic exercise included eleven minutes of aerobic treadmill exercises followed by eleven minutes of ergometer cycling. After warming up for three minutes, the participants underwent a six-minute training followed by a 2 minute cool-down (Fig. 4).

\section{Physical activity evaluation}

Hemophilia Activity List (HAL), with 42 items in 7 domains, was used to assess the physical activity of IWH. Each topic describes hardship caused by hemophilia over the past month. Higher scores indicate better functional status (24). Control and intervention groups were asked to maintain their routine physical activity and diet during the study $(10,25)$. Upon the completion of the six-week intervention, a belated one-month intervention was performed in the control group, which was not followed by any tests.

\section{Joint health status}

The HJHS 2.1 with a total score of 124 was used to assess the joint health status in each participant, with higher scores denoting worse joint health (26). 


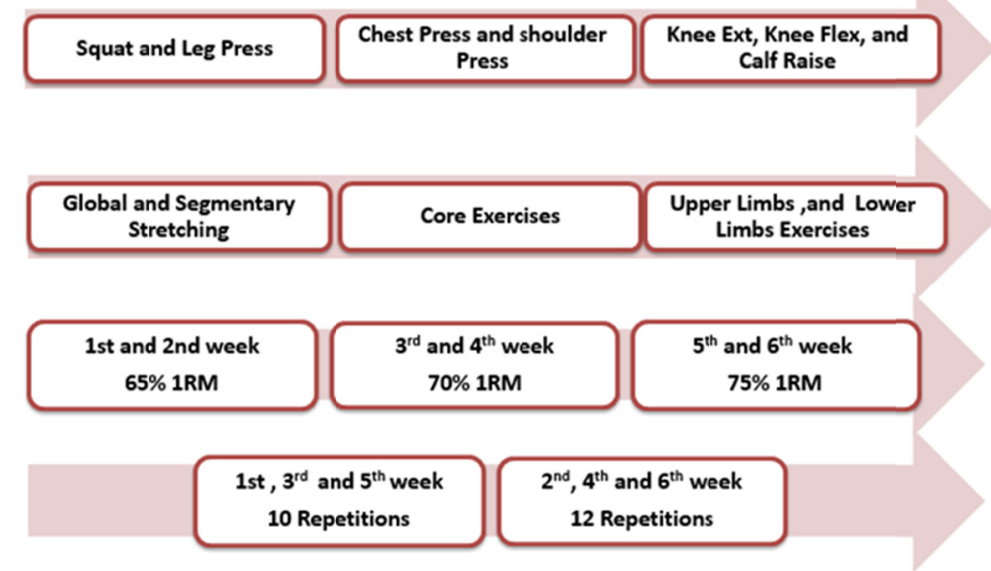

Fig. 2. The exercise protocol in the resistance training group. 1RM, One Repetition Maximum

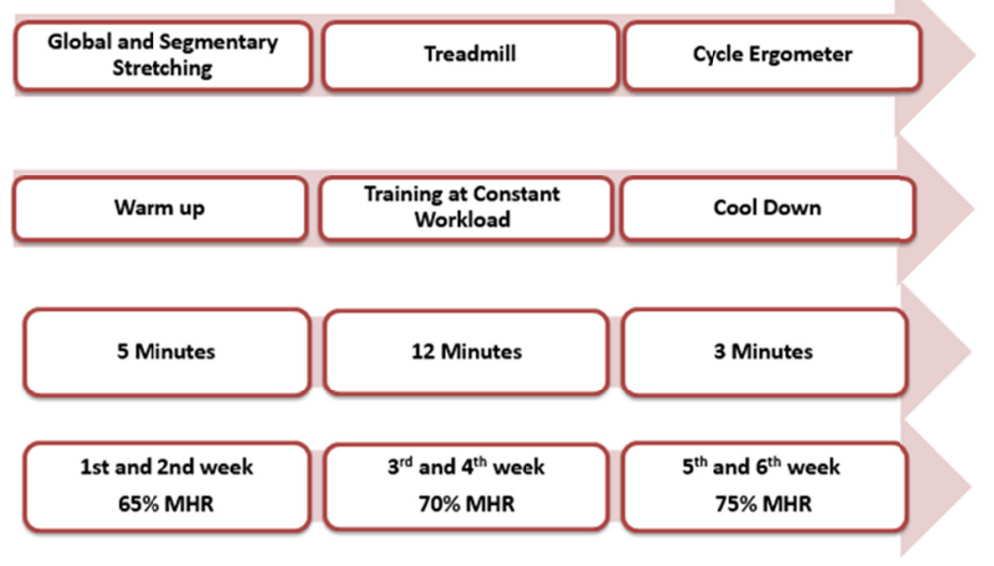

Fig. 3. The exercise protocol in the aerobic training group. MHR, Maximum Heart Rate

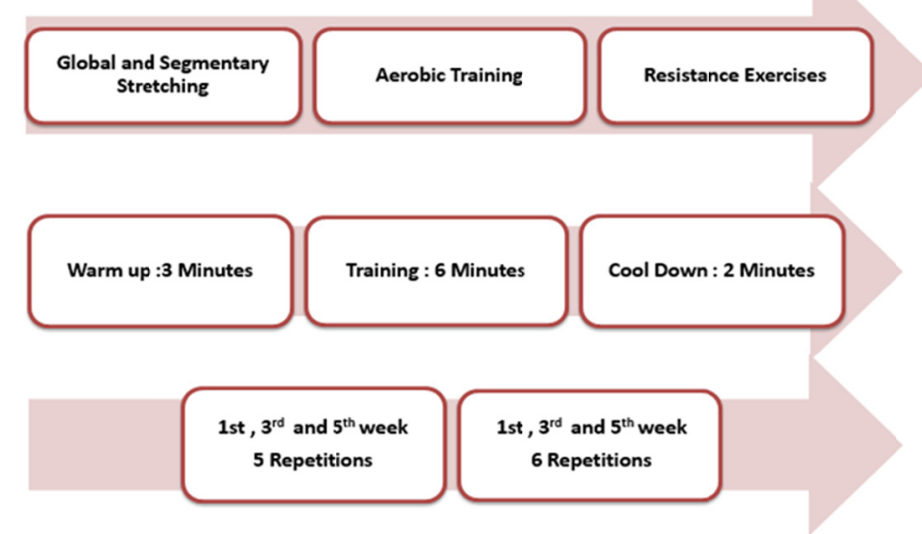

Fig. 4. The exercise protocol in the combined training group

Body fat assessment and anthropometric measurement

At the end of the expiration, a tape measure was used to measure waist circumference (WC) in the thinnest part of the body (10). Also, for measuring the hip circumference (HC), the level of maximal protrusion of the gluteal muscles was selected (27). 


\section{Blood pressure assessment}

SBP and DBP were measured by blood pressure monitor (Omron, BP7250, Japan) in a sitting position after at least 10 minutes of rest before starting exercises and 30 minutes after the end of the training from the left brachial artery, and a mean of the three measurements was obtained (14).

\section{QOL Assessment}

The SF-36 was used to assess QOL before and six weeks after the intervention. It includes eight domains and 36 items that measured bodily pain (BP), social functioning (SF), physical functioning (PF), general health $(\mathrm{GH})$, role physical (RP), mental health $(\mathrm{MH})$, vitality (VT), and role emotional (RE). The physical component score (PCS) subscale comprised the GH, BP, RP, and PF subscales, and the general subscale of the mental component score (MCS) comprised the RE, VT, SF, and MH subscales. A higher score indicated higher levels of QOL (4).

\section{Lipid profiles analysis}

Following $24 \mathrm{~h}$ before the first training session and 48 $\mathrm{h}$ after its last session, fasting blood collection was performed between 8 to 9 AM (13). All blood samples were stored immediately at $-80^{\circ} \mathrm{C}$ before analysis. The serum concentration of HDL, LDL, TG, and TC were measured before and after the six weeks by a photometric method using Pars Azmoon kits, Iran, in the ICHCC laboratory, Tehran, Iran.

\section{Statistical analyses}

The Kolmogorov-Smirnov test was used to confirm the distribution of the data follows a normal one. Furthermore, the differences among the four groups were determined using the univariate analyses of covariance (ANCOVA) and the discrepancies between the data with significant interaction effects using the Bonferroni post hoc test. The treatment group was considered the constant; the preintervention value was taken as the covariate and the postintervention value of the same variable as the dependent variable. All the analyses were performed in IBM SPSS Statistics for Windows, version 20 (IBM Corp., Armonk, N.Y., USA). Furthermore, effect sizes measured using the Cohen d. $\mathrm{P}<0.05$ was set as the level of statistical signifi- cance.

\section{Results}

\section{Anthropometric and hemodynamic parameters} changes

The demographic details of the participants are presented in Table 1. A significant decrease was observed in the $\mathrm{RT}$, AT, and CT groups compared to the control group in terms of weight, BMI, WHR, WC, SBP, and DBP $(p<0.05)$ (Table 2). There was no significant difference among the intervention groups $(\mathrm{p}>0.05)$.

\section{Changes in the total HJHS}

There was no musculoskeletal bleeding in the intervention groups. The total HJHS was significantly reduced in the RT, AT, and CT groups compared to the control group through the six weeks intervention $(\mathrm{p} \leq 0.001)$ (Table 2$)$. There was no significant difference among the intervention groups $(\mathrm{p}>0.05)$.

\section{HAL sum score changes}

A significant increase was observed in the HAL sum score in all the intervention groups compared to the control group after six weeks of intervention $(\mathrm{p} \leq 0.001)$ ble 2). There was no significant difference among the intervention groups $(\mathrm{p}>0.05)$.

\section{Changes in SF-36 subscales}

The study groups were not significantly different in terms of eight subscales of the SF-36 at baseline $(p>0.05)$. The total SF-36 score, MCS, PCS, MH, RE, SF, VT, GH, $\mathrm{BP}$, and $\mathrm{PF}$ were significantly increased through the sixweek intervention in the RT, AT, and CT groups compared to the control group $(\mathrm{p} \leq 0.001)$ (Table 3$)$. No significant difference was observed among the intervention groups $(\mathrm{p}>0.05)$.

\section{Lipid profile changes}

A significant increase in HDL-C was also observed in the AT, RT and CT groups compared to the control group after six weeks $(\mathrm{p}<0.001)$ (Table 4$)$. A significant decrease was observed in the LDL-C and TC in the AT, RT, and CT groups compared to the control group $(p<0.05)$.

Table 1. Subject characteristics at baseline

\begin{tabular}{|c|c|c|c|c|c|}
\hline Characteristic & $\mathrm{RT}(\mathrm{n}=15)$ & $\operatorname{AT}(n=15)$ & $\mathrm{CT}(\mathrm{n}=15)$ & $\mathrm{CON}(\mathrm{n}=15)$ & $\mathrm{p}$ \\
\hline Age(yr) & $46.87(4.27)$ & $46.2(5.59)$ & $46(6.07)$ & $46.017(4.68)$ & 0.967 \\
\hline Height (m) & $1.76(0.03)$ & $1.76(0.03)$ & $1.77(0.04)$ & $1.76(0.04)$ & 0.863 \\
\hline Weight (kg) & $85.13(5.46)$ & $86.33(4.68)$ & $87.73(7.83)$ & $87.07(4.23)$ & 0.865 \\
\hline $\operatorname{BMI}\left(\mathrm{kg} / \mathrm{m}^{2}\right)$ & $27.82(1.08)$ & $27.74(1.12)$ & $28.01(1.31)$ & $27.85(1.05)$ & 0.931 \\
\hline Factor VIII activity $\left(\mathrm{IUdL}^{-1}\right)$ & $3.77(0.74)$ & $4(0.830$ & $3.92(0.84)$ & $3.82(0.66)$ & 0.856 \\
\hline Total HJHS Scores & $6.2(1.2)$ & $5.93(1.03)$ & $7.06(1.86)$ & $6.4 .6(1.4)$ & 0.165 \\
\hline HAL (Sum Score) & $171.6(4.04)$ & $171.4(3.41)$ & $169.7(4.58)$ & $172.66(6.11)$ & 0.391 \\
\hline Total SF-36 & $76.7(6.6)$ & $77.8(8.2)$ & $75.2(4.7)$ & $78.5(6.5)$ & 0.570 \\
\hline HDL-C(mg/dL) & $39.66(2.35)$ & $41.13(2.58)$ & $40.46(3.94)$ & $40.6(3.22)$ & 0.872 \\
\hline LDL-C(mg/dL) & $119.06(16.34)$ & $117.73(13.6)$ & $122.93(15.88)$ & $116.86(14.40$ & 0.949 \\
\hline $\mathrm{TC}(\mathrm{mg} / \mathrm{dL})$ & $168.2(17.1)$ & $173.33(8.85)$ & $172.53(37.07)$ & $176(25.96)$ & 0.906 \\
\hline $\mathrm{TG}(\mathrm{mg} / \mathrm{dL})$ & $149.13(45.8)$ & $135.4(36.8)$ & $155.2(44.61)$ & $146.2(49.23)$ & 0.973 \\
\hline
\end{tabular}

Data are expressed as the mean \pm standard deviation.

RT, resistance training; AT, aerobic training; CT, combined training; CON, control; BMI, Body Mass Index; HJHS, Hemophilia Joint Health Score, HAL; Hemophilia Activity List; SF-36, Short- Form 36; HDL-C, high-density lipoprotein cholesterol; LDL-C, Low-density lipoprotein cholesterol; TC, Total cholesterol; TG, Triglycerides 
Table 2. Anthropometric parameters, blood pressure, Total HJHS and HAL sum score before and after six weeks of training

\begin{tabular}{|c|c|c|c|c|c|c|c|c|c|c|c|c|c|c|}
\hline \multirow[t]{2}{*}{ Variable } & \multicolumn{4}{|c|}{ RT group } & \multicolumn{4}{|c|}{ AT group } & \multicolumn{4}{|c|}{ CT group } & \multicolumn{2}{|c|}{ CON group } \\
\hline & Pre & Post & $\bar{P}$ & Cohen d & Pre & Post & $P$ & Cohen d & Pre & Post & $\bar{P}$ & Cohen d & Pre & Post \\
\hline Weight $(\mathrm{kg})$ & $\begin{array}{l}86.13 \\
(5.46)\end{array}$ & $\begin{array}{c}85.2 \\
(5.37)^{\dagger}\end{array}$ & $\leq 0.001$ & 0.17 & $\begin{array}{l}86.33 \\
(4.68)\end{array}$ & $\begin{array}{c}85.33 \\
(4.92)^{\dagger}\end{array}$ & $\begin{array}{l}\leq \\
0.001\end{array}$ & 0.2 & $\begin{array}{l}87.73 \\
(7.83)\end{array}$ & $\begin{array}{c}86.13 \\
(7.13)^{\dagger}\end{array}$ & $\leq 0.001$ & 0.21 & $87.07(4.23)$ & $\begin{array}{l}87.47 \\
(4.06)\end{array}$ \\
\hline BMI $\left(\mathrm{kg} / \mathrm{m}^{2}\right)$ & $\begin{array}{l}27.82 \\
(1.08)\end{array}$ & $\begin{array}{c}27.56 \\
(1.04)^{\dagger}\end{array}$ & 0.007 & 0.24 & $\begin{array}{l}27.74 \\
(1.12)\end{array}$ & $\begin{array}{c}27.43 \\
(1.22)^{\dagger}\end{array}$ & 0.001 & 0.26 & $\begin{array}{l}28.01 \\
(1.31)\end{array}$ & $\begin{array}{c}27.48 \\
(1.11)^{\dagger}\end{array}$ & $\leq 0.001$ & 0.43 & $27.85(1.05)$ & $\begin{array}{l}27.99 \\
(1.15)\end{array}$ \\
\hline WHR & $\begin{array}{c}0.932 \\
(0.039)\end{array}$ & $\begin{array}{c}0.928 \\
(0.36)^{\dagger}\end{array}$ & 0.006 & 0.17 & $\begin{array}{c}0.928 \\
(0.561)\end{array}$ & $\begin{array}{c}0.925 \\
(0.61)^{\dagger}\end{array}$ & 0.020 & 0.005 & $\begin{array}{c}0.932 \\
(0.048)\end{array}$ & $\begin{array}{c}0.928 \\
(0.047)^{\dagger}\end{array}$ & 0.004 & 0.09 & $0.944(0.039)$ & $\begin{array}{c}0.948 \\
(0.039)\end{array}$ \\
\hline $\mathrm{WC}(\mathrm{Cm})$ & $\begin{array}{l}100.3 \\
(7.48)\end{array}$ & $99.9(7.43)^{\dagger}$ & 0.010 & 0.05 & $99.16(7.7)$ & $\begin{array}{c}98.1 \\
(7.83)^{\dagger}\end{array}$ & 0.048 & 0.13 & $97.6(9.06)$ & $\begin{array}{c}97.1(9.09) \\
\dagger\end{array}$ & 0.005 & 0.05 & $100.66(7.48)$ & $\begin{array}{l}101.06 \\
(7.54)\end{array}$ \\
\hline $\mathrm{HC}(\mathrm{Cm})$ & $\begin{array}{l}106.86 \\
(7.26)\end{array}$ & $106.8(7.33)$ & 0.91 & 0.008 & $\begin{array}{l}106.53 \\
(5.53)\end{array}$ & $\begin{array}{l}106.53 \\
(5.53)\end{array}$ & 1 & 0 & $\begin{array}{c}104.33 \\
(5.2)\end{array}$ & $\begin{array}{c}104.33 \\
(5.2)\end{array}$ & 1 & 0 & $106.26(5.07)$ & $\begin{array}{c}106.26 \\
(5.07)\end{array}$ \\
\hline $\mathrm{SBP}(\mathrm{mm})$ & $\begin{array}{c}130.26 \\
(6.1)\end{array}$ & $122(6.96)^{\dagger}$ & $\leq 0.001$ & 1.26 & $\begin{array}{l}131.13 \\
(4.73)\end{array}$ & $\begin{array}{c}120.7 \\
(8.46)^{\dagger}\end{array}$ & $\leq$ & 1.52 & $\begin{array}{l}130.46 \\
(7.65)\end{array}$ & $\begin{array}{l}120.6 \\
(7.9)^{\dagger}\end{array}$ & $\leq 0.001$ & 1.26 & $125.53(7.63)$ & $\begin{array}{l}127.73 \\
(6.54)\end{array}$ \\
\hline $\mathrm{DBP}(\mathrm{mm})$ & $82.2(6.39)$ & $76.6(5.92)^{\dagger}$ & $\leq 0.001$ & 0.91 & $\begin{array}{l}83.53 \\
(5.61)\end{array}$ & $\begin{array}{c}77.2 \\
(6.32)^{\dagger}\end{array}$ & $\begin{array}{c}\leq \\
0.001\end{array}$ & 1.06 & $83.3(4.59)$ & $\begin{array}{c}72.86 \\
(5.33)^{\dagger}\end{array}$ & $\leq 0.001$ & 2.1 & $79.33(5.42)$ & $\begin{array}{c}82.2 \\
(4.79)\end{array}$ \\
\hline Total HJHS & $6.2(1.2)$ & $\begin{array}{c}3.86 \\
(1.3)^{\dagger}\end{array}$ & $\leq 0.001$ & 1.32 & $\begin{array}{c}5.93 \\
(1.03)\end{array}$ & $\begin{array}{c}4.1 \\
(1.8)^{\dagger}\end{array}$ & $\begin{array}{c}\leq \\
0.001\end{array}$ & 1.25 & $\begin{array}{c}7.06 \\
(1.86)\end{array}$ & $\begin{array}{c}4.2 \\
(1.26)^{\dagger}\end{array}$ & $\leq 0.001$ & 1.81 & $6.46(1.4)$ & $\begin{array}{c}7.6 \\
(1.99)\end{array}$ \\
\hline $\begin{array}{l}\text { HALsum } \\
\text { score }\end{array}$ & $\begin{array}{l}171.6 \\
(4.04)\end{array}$ & $204.6(4.7)^{\dagger}$ & $\leq 0.001$ & 7.3 & $\begin{array}{l}171.4 \\
(3.41)\end{array}$ & $\begin{array}{c}198.8 \\
(5.36)^{\dagger}\end{array}$ & $\begin{array}{c}\leq \\
0.001\end{array}$ & 6.1 & $\begin{array}{l}169.7 \\
(4.58)\end{array}$ & $\begin{array}{c}211.4 \\
(4.53)^{\dagger}\end{array}$ & $\leq 0.001$ & 9.16 & $172.66(6.11)$ & $\begin{array}{l}172.8 \\
(3.9)\end{array}$ \\
\hline
\end{tabular}

Data are expressed as the mean \pm standard deviation.

RT, resistance training; AT, aerobic training; CT, combined training; CON, control; BMI, Body mass index; WHR, waist to hip ratio fat mass ; WC, Waist circumference ; HC, hip circumference, SBP, Systolic Blood Pressure; DBP, Diastolic Blood Pressure; HJHS, Hemophilia Joint Health Score; HAL; Hemophilia Activity List. †ं Significant difference related to the control group. 
Table 3. SF-36 subscales before and after six weeks of training

\begin{tabular}{|c|c|c|c|c|c|c|c|c|c|c|c|c|c|c|}
\hline \multirow[t]{2}{*}{ Variable } & \multicolumn{4}{|c|}{ RT group } & \multicolumn{4}{|c|}{ AT group } & \multicolumn{3}{|c|}{ CT group } & \multicolumn{3}{|c|}{ CON group } \\
\hline & Pre & Post & $\mathrm{P}$ & $\begin{array}{c}\text { Cohen } \\
\text { d }\end{array}$ & Pre & Post & $\mathrm{P}$ & Cohen $\mathrm{d}$ & Pre & Post & $P$ & $\begin{array}{c}\text { Cohen } \\
\text { d }\end{array}$ & Pre & Post \\
\hline PF & $\begin{array}{l}75.73 \\
(6.49)\end{array}$ & $85.6(4.5)^{\dagger}$ & $\leq 0.001$ & 1.75 & $\begin{array}{c}77.26 \\
(5.4)\end{array}$ & $85.9(4.5)^{\dagger}$ & $\leq 0.001$ & 1.73 & $73.8(8.8)$ & $85.8(8.3)^{\dagger}$ & $\leq 0.001$ & 1.4 & $79.2(6.8)$ & $\begin{array}{l}77.9 \\
(6.5)\end{array}$ \\
\hline $\mathrm{RP}$ & $80(6.8)$ & $\begin{array}{c}90.1 \\
(6.03)^{\dagger}\end{array}$ & $\leq 0.001$ & 1.72 & $\begin{array}{c}76.65 \\
(8)\end{array}$ & $85.3(6.5)^{\dagger}$ & $\leq 0.001$ & 1.18 & $80.6(7.3)$ & $92.3(5.8)^{\dagger}$ & $\leq 0.001$ & 1.84 & $82.5(5.8)$ & $\begin{array}{c}78.4 \\
(8.7)\end{array}$ \\
\hline BP & $77.2(6.79)$ & $90(7.58)^{\dagger}$ & $\leq 0.001$ & 1.78 & $\begin{array}{l}77.5 \\
(6.5)\end{array}$ & $82.5(6.2)^{\dagger}$ & $\leq 0.001$ & 0.8 & $78.03(4.2)$ & $92.6(5.9)^{\dagger}$ & $\leq 0.001$ & 2.84 & $80.8(7)$ & $\begin{array}{l}78.3 \\
(7.1)\end{array}$ \\
\hline GH & $76.67(10)$ & $\begin{array}{c}83.75 \\
(7.42)^{\dagger}\end{array}$ & $\leq 0.001$ & 0.8 & $\begin{array}{l}77.9 \\
(8.2)\end{array}$ & $81.8(7)^{\dagger}$ & $\leq 0.001$ & 0.51 & $\begin{array}{l}78.02 \\
(6.9)\end{array}$ & $87(5.4)^{\dagger}$ & $\leq 0.001$ & 1.45 & $81.1(9.9)$ & $\begin{array}{l}77.9 \\
(9.1)\end{array}$ \\
\hline VT & $72.3(8.6)$ & $84.8(6.5)^{\dagger}$ & $\leq 0.001$ & 1.64 & $\begin{array}{l}77.6 \\
(8.4)\end{array}$ & $85.2(6.3)^{\dagger}$ & $\leq 0.001$ & 1.02 & $78(9.6)$ & $90(7)^{\dagger}$ & $\leq 0.001$ & 1.42 & 78.8 (8.9) & $77.6(8)$ \\
\hline SF & $77.86(8.6)$ & $86.1(5.9)^{\dagger}$ & $\leq 0.001$ & 1.11 & $\begin{array}{l}75.6 \\
(7.2)\end{array}$ & $82.5(6.2)^{\dagger}$ & $\leq 0.001$ & 1.02 & 80.6 (12) & $91.5(7.6)^{\dagger}$ & $\leq 0.001$ & 1.08 & $78.9(8)$ & $\begin{array}{l}77.7 \\
(8.5)\end{array}$ \\
\hline $\mathrm{RE}$ & $\begin{array}{c}81.05 \\
(7.57)\end{array}$ & $90.3(6.7)^{\dagger}$ & $\leq 0.001$ & 1.29 & $\begin{array}{l}77.4 \\
(9.5)\end{array}$ & $84.6(8.6)^{\dagger}$ & $\leq 0.001$ & 0.79 & $81.7(10)$ & $91.4(7.3)^{\dagger}$ & $\leq 0.001$ & 1.1 & $82.9(7.9)$ & $\begin{array}{c}81.9 \\
(8.15)\end{array}$ \\
\hline MH & $74.8(6.7)$ & $82.8(4.5)^{\dagger}$ & $\leq 0.001$ & 1.4 & $\begin{array}{l}77.2 \\
(6.5)\end{array}$ & $84(6.7)^{\dagger}$ & $\leq 0.001$ & 1.03 & $79.8(10)$ & $88.8(7.2)^{\dagger}$ & $\leq 0.001$ & 1.03 & $78.6(6.6)$ & $\begin{array}{l}75.7 \\
(5.9)\end{array}$ \\
\hline PCS & 77.4 (3.9) & $87.3(2.8)^{\dagger}$ & $\leq 0.001$ & 2.92 & $\begin{array}{l}78.12 \\
(2.67)\end{array}$ & $85.2(2.8)^{\dagger}$ & $\leq 0.001$ & 2.93 & $77.6(3.06)$ & $89.4(2.9)^{\dagger}$ & $\leq 0.001$ & 3.26 & $80.9(3.3)$ & $\begin{array}{l}78.1 \\
(3.3)\end{array}$ \\
\hline MCS & $76.5(5.6)$ & $86(4.2)^{\dagger}$ & $\leq 0.001$ & 1.92 & $\begin{array}{c}76.97 \\
(4.7)\end{array}$ & $84(4.42)^{\dagger}$ & $\leq 0.001$ & 1.54 & $80(5.5)$ & $90.6(4.4)^{\dagger}$ & $\leq 0.001$ & 2.12 & $79.8(2.72)$ & $\begin{array}{l}78.25 \\
(2.97)\end{array}$ \\
\hline $\begin{array}{c}\text { Total SF- } \\
36\end{array}$ & $76.7(6.6)$ & $87(4.9)^{\dagger}$ & $\leq 0.001$ & 1.77 & $\begin{array}{l}77.8 \\
(8.2) \\
\end{array}$ & $86.6(8.4)^{\dagger}$ & $\leq 0.001$ & 1.06 & $75.2(4.7)$ & $89.8(4.8)^{\dagger}$ & $\leq 0.001$ & 3.07 & $78.5(6.5)$ & $76.7(6)$ \\
\hline
\end{tabular}

Data are expressed as the mean \pm standard deviation.

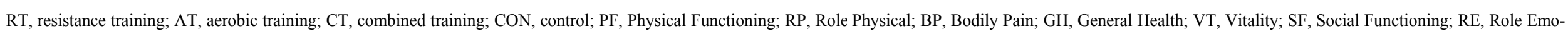
tional; MH, Mental Health; PCS, Physical Component Score; MCS, Mental Component Score

$\dagger$ Significant difference related to the control group. 
Exercise training and lipid profiles in overweight hemophilia

Table 4. Lipid profiles before and after six weeks of training

\begin{tabular}{|c|c|c|c|c|c|c|c|c|c|c|c|c|c|c|}
\hline \multirow{2}{*}{ Variable } & \multicolumn{4}{|c|}{ RT group } & \multicolumn{4}{|c|}{ AT group } & \multicolumn{4}{|c|}{ CT group } & \multicolumn{2}{|c|}{ CON group } \\
\hline & Pre & Post & $P$ & Cohen $\mathrm{d}$ & Pre & Post & $P$ & Cohen $\mathrm{d}$ & Pre & Post & $P$ & Cohen $\mathrm{d}$ & Pre & Post \\
\hline $\begin{array}{l}\text { HDL-C } \\
(\mathrm{mg} / \mathrm{dL})\end{array}$ & $\begin{array}{c}39.66 \\
(2.35)\end{array}$ & $41.46(2.5)^{\dagger}$ & 0.001 & 0.74 & $\begin{array}{l}41.13 \\
(2.58)\end{array}$ & $\begin{array}{c}42.33 \\
(2.35)^{\dagger}\end{array}$ & 0.002 & 0.48 & $\begin{array}{c}40.46 \\
(3.94)\end{array}$ & $\begin{array}{c}42.8 \\
(3.76)^{\dagger}\end{array}$ & $\leq 0.001$ & 0.6 & $\begin{array}{c}40.6 \\
(3.22)\end{array}$ & $\begin{array}{c}39.2 \\
(3.48)\end{array}$ \\
\hline $\begin{array}{l}\text { LDL-C } \\
(\mathrm{mg} / \mathrm{dL})\end{array}$ & $\begin{array}{c}119.06 \\
(16.34)\end{array}$ & $\begin{array}{c}116.4 \\
(15.98) \dagger\end{array}$ & 0.010 & 0.16 & $\begin{array}{l}117.73 \\
(13.6)\end{array}$ & $\begin{array}{c}115.13 \\
(13.2)^{\dagger}\end{array}$ & 0.009 & 0.19 & $\begin{array}{c}122.93 \\
(15.88)\end{array}$ & $\begin{array}{c}117.4 \\
(15.09)^{\dagger}\end{array}$ & $\leq 0.001$ & 0.35 & $\begin{array}{l}116.86 \\
(14.4)\end{array}$ & $\begin{array}{c}119.46 \\
(13.82)\end{array}$ \\
\hline $\mathrm{TC}(\mathrm{mg} / \mathrm{dL})$ & $\begin{array}{c}168.2 \\
(17.1)\end{array}$ & $165(17.01)^{\dagger}$ & 0.009 & 0.18 & $\begin{array}{l}173.33 \\
(38.8)\end{array}$ & $\begin{array}{c}168.5 \\
(36.6)^{\dagger}\end{array}$ & $\leq 0.001$ & 0.12 & $\begin{array}{l}172.53 \\
(37.07)\end{array}$ & $\begin{array}{c}166.6 \\
(37.25)^{\dagger}\end{array}$ & $\leq 0.001$ & 0.15 & $\begin{array}{c}176 \\
(25.96)\end{array}$ & $\begin{array}{r}176.86 \\
(25.74)\end{array}$ \\
\hline $\mathrm{TG}(\mathrm{mg} / \mathrm{dL})$ & $\begin{array}{l}149.13 \\
(45.8)\end{array}$ & $\begin{array}{c}146.33 \\
(45.38)\end{array}$ & 0.070 & 0.06 & $\begin{array}{c}135.4 \\
(36.8)\end{array}$ & $\begin{array}{c}132.6 \\
(35.9)^{\dagger}\end{array}$ & 0.030 & 0.07 & $\begin{array}{c}155.2 \\
(44.61)\end{array}$ & $\begin{array}{c}147.6 \\
(37.51)^{\dagger}\end{array}$ & $\leq 0.001$ & 0.18 & $\begin{array}{c}146.2 \\
(49.23)\end{array}$ & $\begin{array}{c}149.46 \\
(49.81)\end{array}$ \\
\hline
\end{tabular}

RT, resistance training; AT, aerobic training; CT, combined training; CON, control; HDL-C, high-density lipoprotein cholesterol; LDL-C, Low-density lipoprotein cholesterol; TC, Total cholesterol; TG, Triglycerides,

†Significant difference related to the control group. 
A significant decrease was also observed in the TG in the CT and AT groups compared to the control group $(p<0.001)$. No significant differences were observed among the intervention groups ( $\mathrm{p}>0.05)$.

Comparing the mean differences among the four groups

There was no significant difference among the interven- tion groups $(p>0.05)$. The decrease observed in the mean differences in the total HJHS, WC, WHR, BMI, TC, LDL$\mathrm{C}$, weight, and TG was significant in the CT group compared to the RT and AT groups. In comparison with the control group, more significant improvement was observed in the PCS, MCS, HAL sum score, and HDL-C in the CT group compared to the RT and AT groups (Table $5)$.

\begin{tabular}{|c|c|c|c|c|}
\hline Variable & Group & MD & SE & $95 \% \mathrm{CI}$ \\
\hline \multicolumn{5}{|l|}{ Weight } \\
\hline & RT & $-1.38 \dagger$ & 0.32 & -2.26 to -0.49 \\
\hline & AT & $-1.43^{\dagger}$ & 0.32 & -2.32 to -0.55 \\
\hline & CT & $-1.96^{\dagger}$ & 0.32 & -2.84 to -1.08 \\
\hline \multicolumn{5}{|l|}{ BMI } \\
\hline & RT & $-0.39^{\dagger}$ & 0.11 & -0.71 to -0.8 \\
\hline & AT & $-0.46^{\dagger}$ & 0.11 & -0.77 to -0.14 \\
\hline & CT & $-0.66^{\dagger}$ & 0.11 & -0.98 to -0.34 \\
\hline \multicolumn{5}{|l|}{ WHR } \\
\hline & RT & $-0.008^{\dagger}$ & 0.002 & -0.015 to -0.002 \\
\hline & AT & $-0.007 \dagger$ & 0.002 & -0.014 to -0.00 \\
\hline & CT & $-0.009^{\dagger}$ & 0.002 & -0.015 to -0.002 \\
\hline \multicolumn{5}{|l|}{ WC } \\
\hline & RT & $-0.79^{\dagger}$ & 0.24 & -1.45 to -0.14 \\
\hline & AT & $-0.66^{\dagger}$ & 0.24 & -1.32 to -0.004 \\
\hline & CT & $-0.86^{\dagger}$ & 0.24 & -1.52 to -0.19 \\
\hline \multirow[t]{3}{*}{ Total HJHS } & RT & $-3.52^{\dagger}$ & 0.44 & -4.7 to -2.3 \\
\hline & AT & $-3.05^{\dagger}$ & 0.44 & -4.26 to -1.83 \\
\hline & CT & $-3.86 \dagger$ & 0.44 & -5.08 to -2.64 \\
\hline \multicolumn{5}{|l|}{ HAL } \\
\hline \multirow[t]{3}{*}{ Sum Score } & RT & $32.5^{\dagger}$ & 1.19 & 29.23 to 35.77 \\
\hline & AT & $26.4 \dagger$ & 1.19 & 23.57 to 30.1 \\
\hline & CT & $40.6^{\dagger}$ & 1.22 & 37.31 to 43.99 \\
\hline \multicolumn{5}{|l|}{ PCS } \\
\hline & RT & $11.43^{\dagger}$ & 0.81 & 9.2 to 13.66 \\
\hline & $\mathrm{AT}$ & $8.78^{\dagger}$ & 0.79 & 6.6 to 10.9 \\
\hline & CT & $\begin{array}{c}8.18 \\
13.36^{\dagger}\end{array}$ & 0.8 & 11.15 to 15.57 \\
\hline \multicolumn{5}{|l|}{ MCS } \\
\hline & RT & $10.2^{\dagger}$ & 0.75 & 8.1 to 12.16 \\
\hline & $\mathrm{AT}$ & $7.95^{\dagger}$ & 0.74 & 5.9 to 9.9 \\
\hline & CT & $12.23^{\dagger}$ & 0.72 & 10.23 to 14.2 \\
\hline \multicolumn{5}{|l|}{ HDL-C } \\
\hline & RT & $2.99^{\dagger}$ & 0.71 & 1.03 to 4.95 \\
\hline & AT & $2.71^{\dagger}$ & 0.71 & 0.76 to 4.67 \\
\hline & CT & $3.7 \dagger$ & 0.71 & 1.75 to 5.65 \\
\hline \multirow[t]{3}{*}{ LDL-C } & RT & $-5.09^{\dagger}$ & 1.53 & -9.3 to -0.89 \\
\hline & AT & $-5.13^{\dagger}$ & 1.53 & -9.33 to -0.93 \\
\hline & CT & $-7.67 \dagger$ & 1.55 & -11.91 to -3.42 \\
\hline \multirow[t]{3}{*}{$\mathrm{TC}$} & $\mathrm{RT}$ & $-4.29 \dagger$ & 1.27 & -7.79 to -0.79 \\
\hline & AT & $-5.74 \dagger$ & 1.27 & -9.23 to -2.25 \\
\hline & CT & $-6.9^{\dagger}$ & 1.27 & -10.38 to -3.41 \\
\hline \multirow[t]{3}{*}{ TG } & RT & -5.91 & 2.28 & -12.15 to 0.32 \\
\hline & AT & $-6.62 \dagger$ & 2.3 & -12.88 to -0.35 \\
\hline & CT & $-10.4 \dagger$ & 2.28 & -16.66 to- 4.14 \\
\hline
\end{tabular}

RT, resistance training; AT, aerobic training; CT, combined training; BMI, Body mass index; WHR, waist to hip ratio; WC, Waist circumference ; HJHS, Hemophilia Joint Health Score; HAL, Hemophilia Activity List; PCS, Physical Component Score; MCS, Mental Component Score; HDL-C, high-density lipoprotein cholesterol; LDL-C, Low-density lipoprotein cholesterol; TC, Total cholesterol; TG, Triglyceride; MD, Mean Difference; SE, Standard Error; CI, Confidence Interval for difference, †The mean difference is significant at the 0.05 llevel. 


\section{Discussion}

The most important finding of this study was a further decrease in LDL-C, TC, and TG and a further increase in the QOL and HDL-C in the CT group compared to the RT and AT groups. Given that no similar studies have been yet conducted on modifying lipid profile using exercise training in IWH, and therefore little information was available for determining the training protocol, this preliminary study was conducted to adapt a training program compatible with musculoskeletal conditions of IWH.

\section{Anthropometric measures}

Significant decrease was observed after six weeks in BMI, WHR, WC, and weight in the CT, RT, and AT groups compared to the control group. Despite the short duration of the study, the designed protocol of the exercises was effective in reducing body weight and improving anthropometric parameters in addition to being safe and not causing musculoskeletal bleeding in the IWH. The tendency for reduction in WHR and WC in the CT, RT and AT groups and a tendency for an increase in WHR and $\mathrm{WC}$ in the control group suggest the preventive and improving effect of AT, RT, and CT for modifying the cardiovascular risk factors in the over-weighted IWH. Henrad et al. (28) measured a high mean factor VIII recovery in inactive IWH suffering over weight, and high BMI, and fat mass index. Given the reductions in weight and BMI in all three types of exercise and its further reduction in the CT group, the present study revealed the potential role of these exercises in improving homeostasis and normalization of factor VIII recovery in IWH. The factor VIII dose is determined by body weight. Therefore, weight control can reduce factor VIII consumption and treatment costs (28). In line with a previous study in overweight and obese subjects (29), the present study confirmed that combined exercises are the more beneficial training approach for improving cardiorespiratory fitness and reducing body weight compared to AT and RT in people suffering from moderate hemophilia A. Thus, CT is recommended to be prescribed for alleviating cardiovascular risk factors and reducing adiposity in obese or overweight IWH.

\section{Hemophilia activity list sum and hemophilia joint health score}

More significant improvements were also observed in the HAL sum score and total HJHS in the CT group compared to in the RT and AT groups. According to BiereRafi et al. (30), lower (worse) HAL sum scores in obese IWH compared to that in normal-weight subjects goes back to the lower limb impaired function.

\section{Quality of Life}

An study showed that those IWHs, who receive prophylaxis, have a better QOL in terms of BP, PF, GH, and MH than those who receive the factor on demand (31). Exercises enhance self-confidence, social adaptations, insight, and healthy body image and help with overcoming disabilities, health problems, and displaying abilities (32). In this study, we administered a prophylactic injection of factor VIII to motivate the patients to participate in exercise training, which had a positive psychological effect on their QOL. It should be noted that the control group also received a prophylactic dose, but their QOL was reduced despite the injection. The present study pioneered the investigation of the effect of AT, RT, and CT on QOL in overweight IWMHA using the SF-36. After six weeks, significant increases were observed in MCS and PCS in all three exercise groups compared to the controls. The improvement observed in the CT group was more significant than in the RT and AT groups. The results of a recent study on the QOL of Greek adults with hemophilia using SF-36 showed that IWH had a lower QOL in all SF-36 subscales, mainly RP, BP, and GH than the healthy population (33). A score difference above minimally important difference (MID) is considered as clinically significant MIDs were also reported for MCS (3) and PCS (2-3) (34). The present study found variations in PCS and MCS in the $\mathrm{AT}$, RT, and CT groups to be different by over eight points, suggesting the decisive role of exercise training in improving the QOL of clinically-overweight IWH. Due to the high cost of coagulation factors, attention to safe, nonpharmaceutical, complementary therapies that have no side effects, such as exercise training, is essential in improving the QOL of these patients.

\section{Blood pressure and lipid profile measures}

Recent reviews and meta-analyses have found aerobic and resistance exercises to significantly reduce blood pressure by approximately $3-4 \mathrm{mmHg}$ in terms of SBP and DBP (14). In the present study, SBP and DBP decreased in all the training groups. HDL-C is a significant, consistent, and independent predictor of CVD. LDL-C can be protected against oxidative damage by HDL-C, which prevents the production of oxidized LDL-C. In the US, the risk of coronary artery disease was increased by $2-3 \%$ through a decrease of $1 \mathrm{mg} / \mathrm{dl}$ in HDL-C (35). In line with previous studies, the present study reported an increase in HDL-C in all the training groups $(9,11,15)$. Banz et al. (36) reported a 13\% increase in HDL-C following 40 minutes of AT on a skiing-style exercise machine at $85 \%$ of $\mathrm{HR}_{\max }$ three times a week.

The risk of atherosclerosis can increase by the accumulation of mild-to-moderate concentrations $(2-10 \mathrm{mmol} / \mathrm{l})$ of TG on arterial walls (15). The present research found a more significant decrease in TG in the CT group compared to the control group. Lemura et al.(15) reported significant reductions in TG and increases in HDL-C after 30 minutes of $\mathrm{AT}$ at $70-75 \%$ of $\mathrm{HR}_{\max }$ three times a week progressed by a 45 -minutes exercise at $85 \%$ of $\mathrm{HR}_{\max }$ four times a week for a total of eight weeks.

The risk of heart diseases can be increased by LDL-C that transports cholesterol to different body cells and leaves its excess on artery walls (11). In a study, the incidence of major vascular events is decreased by approximately $5 \%$ by a decrease of one $\mathrm{mmol} / \mathrm{l}$ in LDL concentrations (37). The present study found significant reductions in LDL-C in the CT, AT, and RT groups compared to the controls. As a combination of lipids, TC is used for generating energy through exercises with specific dura- 
tion and intensity. Elevated TC is a significant risk factor for CVD (37). In our study, the most significant reduction in TC was observed in the CT group compared to the controls. Ha and So (17) investigated a combination of 30 minutes AT at $60-80 \%$ of $\mathrm{HR}_{\max }$ with 30 minutes of RT at 12-15 RM for 12 weeks in 16 participants aged 2026 years. They reported improvements in lipid profile during the exercise along with reductions in TC, LDL-C, and $\mathrm{TG}$, although the changes were statistically insignificant compared to the corresponding figures in the control group. Tokudome et al. (19) reported a significant reduction of $8 \%$ in TG, a significant increase of $3.5 \%$ in HDL-C, and no significant changes in LDL and TC in older Japanese adults after twelve weeks of home-based combined RT and walking.

Increasing the activity of lipoprotein lipase (LPL) in mobilizing fatty tissues and converting them to free fatty acids for energy generation and reductions in hepatic TG synthase constitute the main mechanisms contributing to improving lipid profiles after strength-endurance exercises (38). The present findings showed that well-designed and supervised CT can influence the levels of blood lipid profiles, most especially HDL-C. The current data suggest that, compared to aerobic or resistance exercise individually, the burden of cardiovascular risk factors can be more significantly reduced in at-risk middle-aged IWH through simultaneous aerobic and resistance training. The main limitations of the present study included a short duration of exercise protocols and a small sample. Another limitation of the study was that the study participants were not blind. Other limitations included failure to control sleep, nutrition, mental status, and physical activity after training sessions. Due to the high cost of coagulation factors and difficulty travelling to the exercise investigation unit, it was not possible to follow up on the study variables. Furthermore, we had limitations in increased weight of dumbbells, barbells, and weights; and increased intensity of aerobic exercise due to patients' low tolerance level and increased risk of spontaneous musculoskeletal bleeding. The effect of aerobic, resistance, and combined exercises needs to be investigated on lipid profiles of IWH with Factor VIII inhibitors, as well.

\section{Conclusion}

The main clinical finding of the present research was the desirable changes in the blood lipid profile and QOL in IWH using all types of exercise. More importantly, the most significant changes were induced in all blood lipid parameters by CT. Therefore, CT may be recommended as a possibly effective therapeutic modality to improve QOL and prevent cardiovascular complications in PWH.

\section{Acknowledgments}

The authors would like to express their gratitude to the participating IWH and ICHCC for their cooperation.

\section{Conflict of Interests}

The authors declare that they have no competing interests.

\section{References}

1. Rodriguez-Merchan EC. Musculoskeletal complications of hemophilia. HSS J. 2010;6(1):37-42:.

2. Rizwan I, Minuk L, Jackson S, Iorio A. Cardiovascular disease prevalence and relevance in haemophilia: a scoping review. Haemophilia. 2015;21:156-166.

3. Konkle BA. The aging patient with hemophilia. Am J Hematol. 2012;87:27-32

4. Walsh M, Macgregor D, Stuckless S, Barrett B, Kawaja M, Scully MF. Health-related quality of life in a cohort of adult patients with mild hemophilia A. J Thromb Haemost. 2008;6:755-61.

5. Soucie JM, Wang C, Siddiqi A, Kulkarni R, Recht M, Konkle BA, et al. The longitudinal effect of bodly adiposity on joint mobility in young males with Haemophilia A. Haemophilia. 2011;17:196-203.

6. Mondal S, Mukhopadhyay SK. Effect of central obesity on lipid profile in healthy young adults. Med J DY Patil Vidyapeeth. 2018;11:152-7.

7. Shaw I, Shaw BS, Krasilshchikov O. Comparison of aerobic and combined aerobic and resistance training on low-density lipoprotein cholesterol concentrations in men. Cardiovasc J Afr. 2009;20(5):290295.

8. Aljabri KS, Bokhari SA, Akl A. The relation between overweight, obesity and plasma lipids in Saudi adults with type 2 diabetes. J Health Spec. 2016;4:140-5.

9. Mann S, Beedie C, Jimenez A. Differential effects of aerobic exercise, resistance training and combined exercise modalities on cholesterol and the lipid profile: review, synthesis and recommendations. Sports Med. 2014;44(2):211-221.

10. Parhampour B, Dadgoo M, Vasaghi- Gharamaleki B, Torkaman G, Ravanbod R, Mirzaii-Dizgah I, et al. The effects of six-week resistance, aerobic and combined exercises on the pro-inflammatory and anti-inflammatory markers in overweight patients with moderate haemophilia A: A randomized controlled trial. Haemophilia. 2019;00:1-10.

11. Vatani SD, Ahmadi S, Ahmadi Dehrashid K, Gharibi F. Changes in cardiovascular risk factors and inflammatory markers of young, healthy, men after six weeks of moderate or high intensity resistance training. J Sports Med Phys Fit. 2011;51(4):695-700.

12. Tambalis K, Panagiotakos DB, Kavouras SA, \& Sidossis LS. Responses of Blood Lipids to Aerobic, Resistance, and Combined Aerobic With Resistance Exercise Training: A Systematic Review of Current Evidence. Angiology. 2009;60(5):614-632.

13. Azarbayjani MA, Abedi B, Peeri M, Rassaee M, Stannard S. Effects of combined aerobic and resistant training on lipid profile and glycemic control in sedentary men. Int Med J. 2014;21:132-136.

14. Schroeder EC, Franke WD, Sharp RL, Lee DC. Comparative effectiveness of aerobic, resistance and combined training on cardiovascular disease risk factors: A randomized controlled trial. Plos One. 2019;7:14.

15. LeMura L, von Duvillard S, Andreacci J, Chelland SA, Russo J. Lipid and lipo- protein profiles, cardiovascular fitness, body composition, and diet during and after resistance, aerobic and combination training in young women. Eur J Appl Physiol. 2000;82:451-8.

16. Leon AS, Sanchez OA. Response of blood lipids to exercise training alone or combined with dietary intervention. Med Sci Sports Exerc. 2001;33:S502-515.

17. Ha CH, So WY. Effects of combined exercise training on body composition and metabolic syndrome factors. Iran J Public Health. 2012;41(8):20-6.

18. Ajayi-Vincent OB, Adesina MO. Effects of resistance training on the blood lipid variables of young adults. Eur Sci J. 2013;9:12.

19. Tokudome M, Nagasaki M, Shimaoka K, Sato Y. Effects of homebased combined resistance training and wal. king on metabolic profiles in elderly Japanese. Geriatr Gerontol Int. 2004;4:157-162.

20. Parhampour B, Torkaman G, Hoorfar H, Hedayati M, Ravanbod R. Effects of the short-term resistance training and pulsed electromagnetic fields on bone metabolism and joint function in severe haemophilia A patients with osteoporosis: a randomized controlled trial. Clin Rehabil. 2014;28:440-450.

21. American College of Sports Medicine. American College of Sports Medicine position stand. Progression models in resistance training for healthy adults. Med Sci Sports Exerc. 2009;41:687-708.

22. Westcott W. ACSM strength training guidelines: role in body 
composition and health enhancement. ACSMs Health Fit J. 2009; 13:14-22.

23. Al-Sharif FA, Al-Jiffri OH, Abd El-Kader SM, Ashmawy EM. Impact of mild versus moderate intensity aerobic walking exercise training on markers of bone metabolism and hand grip strength in moderate hemophilic A patients. Afr Health Sci. 2014;14:11-16.

24. van Genderen FR Westers P, Heijnen L, de Kleijn P, van den Berg HM, Helders PJ, et al. Measuring patients' perceptions on their functional abilities: validation of the Haemophilia Activities List. Haemophilia. 2006;12:36-46.

25. Donges CE, Duffield R, Guelfi KJ, Smith GC, Adams DR, Edge JA. Comparative effects of single-mode vs. duration-matched concurrent exercise training on body composition, low-grade inflammation, and glucose regulation in sedentary, overweight, middle-aged men. Appl Physiol Nutr Metab. 2013;38:779-788.

26. Sluiter D, Foppen W, de Kleijn P, Fischer K. Haemophilia Joint Health Score in healthy adults playing sports. Haemophilia. 2014;20:282-286

27. Jorge ML, de Oliveira VN, Resende NM, Paraiso LF, Calixto A, Diniz ALD, et al. The effects of aerobic, resistance, and combined exercise on metabolic control, inflammatory markers, adipocytokines, and muscle insulin signaling in patients with type 2 diabetes mellitus. Metabolism. 2011;60:1244-1252.

28. Henrard S, Speybroeck N, Hermans C. Body weight and fat mass index as strong predictors of factor VIII in vivo recovery in adults with hemophilia A. J Thromb Haemost. 2011;9(9):1784-90.

29. Ho SS, Dhaliwal SS, Hills AP, Pal S. The effect of 12 weeks of aerobic, resistance or combination exercise training on cardiovascular risk factors in the overweight and obese in a randomized trial. BMC Public Health. 2012;12:704.

30. Biere-Rafi S, Haak BW, Peters M, Gerdes VE, Büller HR, Kamphuisen PW. The impairment in daily life of obese haemophiliacs. Haemophilia. 2011;17:204-208.

31. Hartl H.K, Reitter S, Eidher U, Ramschak H, Ay C, Pabinger I. The impact of severe haemophilia on the social status and quality of life among Austrian haemophiliacs. Haemophilia. 2008;14:703-8.

32. Von Mackensen S. Quality of life and sports activities in patients with haemophilia. Haemophilia 2007;13(2):38-43.

33. Varaklioti A, Kontodimopoulos N, Niakas D, Kouramba A, Katsarou O. Health-Related Quality of Life and Association With Arthropathy in Greek Patients with Hemophilia. Clin Appl Thromb Hemost. 2018;24:815-821.

34. St-Louis J, Urajnik DJ , Ménard F , Cloutier S, Klaassen RJ , Ritchie $\mathrm{B}$, et al. Generic and disease -specific quality of life among youth and young men with hemophilia in canada. BMC Hematol. 2016;16:13

35. Mavi M. Effect of aerobic exercise on blood lipid levels in postmenopausal women. Univ Med. 2009;28:17-24.

36. Banz WJ, Maher MA, Thompson WG, Bassett DR, Moore W, Ashraf M, et al. Effects of resistance versus aerobic training on coronary artery disease risk factors. Exp Biol Med (Maywood). 2003;228(4):434-40.

37. Rossi FE, Fortaleza ACS, Neves LM, Diniz TA, de Castro MR, Buonani $\mathrm{C}$, et al. Combined training (strength plus aerobic) potentiates a reduction in body fat but only functional training reduced lowdensity lipoprotein cholesterol in postmenopausal women with a similar training load. J Exerc Rehabil. 2017;13(3):322-329.

38. Sedaghat H, Fattahi Bafghi A, Emami A. The Effect of Combined Exercise Training Course with Curcumin Supplementation on Lipid Profiles of Inactive Middle-Aged Men. Iran J Diabetes Obes. 2018;10(2):61-66 